\title{
Dengue Co-Infection Microfilaria Presenting with Intestinal Obstruction: A Case Report
}

\author{
A KUMAR $^{\mathrm{a}}, \mathrm{S} \mathrm{MANCHANDA}^{\mathrm{b}}$, PK PANDA $^{\mathrm{c}}$, M JAIN $^{\mathrm{d}}, \mathrm{V} \mathrm{KUMAR}^{\mathrm{d}}$, A BISWAS $^{\mathrm{f}}$
}

\begin{abstract}
Summary:
In the recent years morbidity caused by dengue epidemic has been devastating. Confection of dengue malaria and filaria has been reported in literature where in filarial antigen was detected in the patient. Concurrent infection by dengue and filaria with high parsetemic microfilariae load in a single individual is very rarely known. The varied clinical profile in dengue is multifactorial and concurrent co-infection may be one of them. Here in this case of concurrent infection with dengue and filarial, the patient presented with intestinal obstruction which responded
\end{abstract}

\section{Introduction:}

Dengue is a serious global public health problem, with 2.5 billion people at risk and an annual range of 50 to 390 million infections.Between 2006 and 2012, around 20,474 dengue cases reported annually in India. Morbidity and mortality caused by dengue is also an issue of great concern. In a recent study from Philippines(2016)mortality related to dengue deaths were found to be around $3 \%$. Coinfection of dengue, malaria and filaria has been reported inliterature where in filarial antigen was detected in the patient ${ }^{1}$.

a. Dr. A Kumar, Assistant Professor, Department of Medicine, All India Institute of Medical Sciences, New Delhi.

b. Dr. S Manchanda, Assistant Professor, Department of Radiodiagnosis, All India Institute of Medical Sciences, New Delhi.

c. Dr. PK Panda, Department of Medicine, All India Institute of Medical Sciences, New Delhi.

d. Dr. M Jain, Department of Medicine, All India Institute of Medical Sciences, New Delhi.

e. Dr. V Kumar, Department of Medicine, All India Institute of Medical Sciences, New Delhi.

f. Prof. Ashutosh Biswas, Professor, Department of Medicine, All India Institute of Medical Sciences, New Delhi.

Address of Correspondence: Dr. Ashutosh Biswas, Professor, Department of Medicine, All India Institute of Medical Sciences, New Delhi, Cell phone; +919868397248, E-mail: asuaiims@gmail.com, drashutoshbiswas@gmail.com

Received: 20 May, 2017

Accepted: 30 September, 2018 dramatically with diethylcarbamizine while other clinicalsyndrome took a long time. Furthermore, if single vector can harbour both the infectious agent, the etiopathogenesis may completely take a different turn and at times project alarming condition.

Key words: Dengue, Microfilaria, Intestinal obstruction, dengue hepatitis.

(J Bangladesh Coll Phys Surg 2019; 37: 35-38)

DOI: http://dx.doi.org/10.3329/jbcps.v37i1.39290

Concurrent infection by dengue and filaria with high parsetemicmicro-filarialload in a single individual is rarely known. Furthermore, pathogenesis of this coinfection is not much known in the literature. It can present with a very challenging clinical profile to medical professionals.Filaria is chronic infection while dengue is an acute infection; so, in filarial endemic areas causality of many atypical and severe dengue cases might go unnoticed. We present here a case of dengue and microfilaria co-infection presenting as intestinal obstruction which is not known in literature and once reviewed, proved an alarming index case scenario and raises a great entomological aspect.

\section{Case report:}

A 20-year-old man resident of central India presented with 20 days history of dry cough with three-day illness of fever, abdominal distension and pain and two episodes of vomiting. On examination, he had mild icterus. Vitals were normal. There was no evidence of lymphangitis, lymphedema, or acute adeno lymphangitis. On systemic examination, there were rhonchi on chest auscultation, distended abdomen with increase bowel sounds on auscultation, suggestive of intestinal obstruction. His relevant lab reports have been tabulated in Table 1. 
Table-I

Investigation details of reported case

\begin{tabular}{|c|c|c|c|c|}
\hline Parameters/Test & Day 1 & Day 4 & Day 8 & Day 15 \\
\hline Haemoglobin(gm/dl) & 17.4 & 13.8 & 12.4 & 12.2 \\
\hline WBC $\left(\right.$ cells $\left./ \mathrm{mm}^{3}\right)$ & $23.5 \times 10^{3}$ & $43 \times 10^{3}$ & $38 \times 10^{3}$ & $9.3 \times 10^{3}$ \\
\hline Platelets $\left(\right.$ cells $\left./ \mathrm{mm}^{3}\right)$ & $13 \times 10^{3}$ & $14 \times 10^{3}$ & $75 \times 10^{3}$ & $130 \times 10^{3}$ \\
\hline Haematocrit (\%) & 52 & 43 & 38 & 38 \\
\hline $\mathrm{AEC}$ & 1504 & 515 & 404 & 558 \\
\hline SGOT(IU/L) & 1011 & 807 & 241 & 163 \\
\hline SGPT(IU/L) & 467 & 370 & 216 & 153 \\
\hline Bilirubin(mg/dl) & 3 & 2.3 & 2.9 & 1.8 \\
\hline ALP(IU/L) & 654 & 599 & 751 & 400 \\
\hline $\operatorname{Albumin}(\mathrm{gm} / \mathrm{dl})$ & 3 & 3.1 & 3 & 3 \\
\hline Urea(mg/dl) & 51 & 19 & 10 & 22 \\
\hline Creatinine $(\mathrm{mg} / \mathrm{dl})$ & 0.2 & 0.4 & 0.2 & 0.3 \\
\hline Dengue Ns1 & $-\mathrm{VE}$ & & & \\
\hline IgM for Dengue & - & + & + & \\
\hline $\mathrm{HBs} \mathrm{Ag}$ & - & - & - & \\
\hline Ani-HCV & - & - & - & \\
\hline IgM for Typhoid & - & - & - & \\
\hline Malarial Ag & - & - & - & \\
\hline PT-INR & 1.3 & 1.5 & 1.2 & 1.1 \\
\hline
\end{tabular}

IU/L-International units per litre; $(+)=$ Positive; $(-)=$ Negative .

His x-ray erect (Figure 1.) abdomen depicted multiple air fluid levels suggestive of intestinal obstruction with mentioned symptomatology above. Ultrasound abdomen was suggestive of gall bladder wall oedema with mild ascites and bilateral pleural effusion. He was

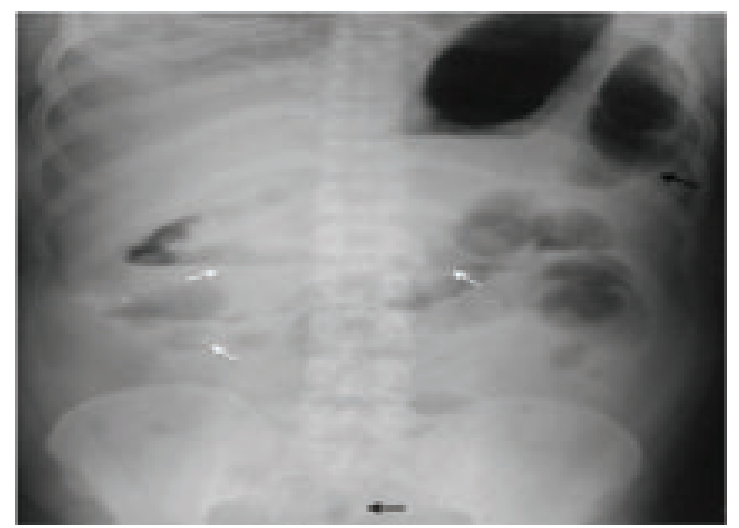

Fig.-1: Plain radiograph of the abdomen (erect position) reveals few small air-fluid levels (white arrows) predominantly in the mid abdomen. Note that there is presence of air in the large bowel (black arrows). tested IgM for dengue by Elisa to be positive on day seven of his total duration of illness. He was tested negative for malaria, typhoid, hepatitis B and hepatitis C. His blood peripheral smear was surprisingly demonstrated high parasetemic load of microfilaria of Wuchereria Bancroft.

Findings to be noted are leucocytosis, eosinophilia, polyserositis, dengue hepatitis (jaundice, transaminitismore than 20 times), respiratory wheeze, long term hospital stay, (admitted for almost 15 days for him to be discharged), slow response to treatment.He was managed conservatively with initially given bowel rest; keeping him nil per orally and gradually built up from liquid to semisolid to normal diet, adequate fluid resuscitation and diethylcarbamazine. Apart from symptoms of intestinal obstruction which showed a dramatic response with diethylcarbamazine in initial days;rest of the clinical syndrome needed prolonged admission. The final diagnosis was microfilaremia and severe dengue infection with multiorgan involvement and dengue hepatitis. 


\section{Discussion:}

Dengue has emerged as a rapidly growing and widespread public health problem in the past two decades, with more than half of the world's population and countries at risk $^{2}$. Our caseseems the first clinical case of dengue with warning signs which turned out to be a case of intestinal obstruction.

Clinical symptomatology of dry cough for last 20 days, abdominalpain along with little air fluid level on abdominal x-ray needed more explanation and insight. Dengue often present with transaminitis but high level of enzymes is rare in classical dengue fever without shock $^{3,4}$. Jaundice is also not frequently present in dengue fever. Dry cough in the preface for 20 days and respiratorywheeze on clinical examination may be contributed to the eosinophilia as secondary to high blood load of microfilaria of WuchereriaBancrofti which seems responded with diethylcarbamazine therapy.It is observed that dengue virus-reactive $T$ cellsactas a effector in the development of dengue hemorrhagic fever (DHF) ${ }^{5}$. The major immunological feature of lymphatic filariasis is an antigen determinedT-helper cells type 2 (Th2) response and IL-10 producing CD4 (+) $\mathrm{T}$ cells with associated decreased Th1 response. This decreased response of T-cell appears to be responsible for the sustained infection often with high densities ofparasite ${ }^{6}$. So, dengue viral load may be high in filarial co-infection case, which probably is responsible for more severe infestation of dengue. We can only presume at this point of time that these atypical findings along with intestinal obstruction and slow recovery may be contributed to the complex immune mediated prolongedinflammation and severe inflammation (bowel oedema with lymphedema) caused by high microfilarial load along with capillary leak syndrome and haemoconcentration caused by dengue coinfection ${ }^{7}$.

Concurrent infectionswith two shown infectious agents may have an overlappingand or atypical syndrome, giving rise to a condition where treatment may need caution and individualised approach. So, while dealing with severe casesin dengue high suspicion should be kept for other co infection; and filarial should be an important consideration especially in filarial endemic zones $^{8}$.

While reviewing very interestingfact came up which makes this case scenario very significant for future research. Concurrent feed of microfilariae (mf) and arboviruses by mosquitoes can enhance the transmission of virus compared with when virus is ingested alone by decreasing external incubation period of dengue to half due to a proven process called as microfilarial enhancement of arboviral transmission ${ }^{7}$, ${ }^{8}$. Though the endemic zones of dengue ${ }^{9}$ and filaria ${ }^{10}$ have some overlap but still with some distinction in India. Aedes can be vector for W. Bancrofti in the given geographical distribution ${ }^{11}$ and it is rationale to presume that it can ingest both the microfilarie and dengue virus if feeds on co-infected person like this case.

\section{Conclusion:}

Filarial co-infection can be a risk factor of severity in dengue. High prevalence of severe dengue may be observed in a filarial endemic zone due to combination of lymphatic channel involvement and capillary leakage.Polyserositis, multi-organ involvement and hypotension are severe manifestation in dengue, however intestinal obstruction is a rare manifestation of dengue which in our case was due to filarial coinfection. Severe and atypical dengue cases should be worked for microfilaria also specially in filarial endemic zones. In such cases if intervened early withdiethylcarbamazine and proper symptomatic management for dengue, a due course of morbidity and mortality can be prevented as was in this case; which otherwise would further add into increase overall mortality with dengue related illness. The spread of dengue in an endemic zone of filariae could be faster as extrinsic incubation period of dengue is decreased to half in Aedes mosquito in presence of microfilarial parasites.

\section{References:}

1. Satyawali V, Pandey S, Rawat V, Khalil M. Triple Co-infection of Malaria, Filaria, and Dengue: A Rare Entity. J Lab Physicians. 2014 Jul;6(2):136-7.

2. Wilder-Smith A, Byass P. The elusive global burden of dengue. Lancet Infect Dis. 2016 Feb 10;

3. Bowman S, Salgado C, DeWaay DJ. Dengue fever presenting with hepatitis. Am J Med Sci. 2012 Oct;344(4):335-6. 
4. Dolo H, Coulibaly YI, Dembele B, Konate S, Coulibaly SY, Doumbia SS, et al. Filariasis Attenuates Anemia and Proinflammatory Responses Associated with Clinical Malaria: A Matched Prospective Study in Children and Young Adults. PLOS Negl Trop Dis. 2012 Nov 1;6(11):e1890.

5. Rothman AL. Immunology and immunopathogenesis of dengue disease. Adv Virus Res. 2003;60:397-419.

6. Babu S, Nutman TB. Immunology of lymphatic filariasis. Parasite Immunol. 2014Aug;36(8):338-46.

7. Vaughan JA, Focks DA, Turell MJ. Simulation models examining the effect of Brugian filariasis on dengue epidemics. Am J Trop Med Hyg. 2009 Jan;80(1):44-50.
8. Gomez-Machorro C, Bennett KE, del Lourdes Munoz M, Black WC. Quantitative trait loci affecting dengue midgut infection barriers in an advanced intercross line of Aedes aegypti. Insect Mol Biol. 2004 Dec;13(6):637-48.

9. Current status of dengue and chinkungunya in India).indd seajphv3n1p22.pdf [Internet]. [cited 2016 Mar 4].

10. Sabesan S, Vanamail P, Raju K., Raju P. Lymphatic filariasis in India: Epidemiology and control measures. J Postgrad Med. 2010;56(3):232.

11. parasites/lymphaticfilariasis/biology_w_bancrofti.html [Internet]. [cited 2016 Mar 4]. 